\title{
Active vibration control of a flexible plate via active force control strategy
}

\begin{abstract}
Active Vibration Control (AVC) is well known nowadays as an optimum technique in vibration suppression of flexible structures. Due to the complexity of the dynamics system of flexible structures, vibration control process is quite a challenge. In this paper, the vibration control of flexible structures using Active Force Control (AFC) method is studied, experimentally. The AVC-AFC controller design is implemented to a full clamped flexible plate system to evaluate its vibration attenuation performance. The system's dynamic model considering the collocated placement of the sensor and actuator is derived within the LabVIEW environment. The first five frequencies of vibration mode were obtained. The result indicated that the AVC-AFC possessed the ability to attenuate vibration of the flexible structure.
\end{abstract}

Keyword: Active force control (AFC); Active vibration control (AVC); Flexible plate; Piezoelectric 